\title{
INCLUSIÓN DE LA ÉTICA Y BIOÉTICA EN LA FORMACIÓN DE PRE Y POSGRADO DEL CIRUJANO-DENTISTA EN PERÚ
}

\author{
Carmen Rosa García Rupaya*
}

\begin{abstract}
Resumen: Se revisan aspectos de la inclusión de la ética y bioética en la formación de pre y posgrado del cirujano-dentista en Perú. Desde el punto de vista de la formación del docente, se analiza la presencia de normatividad ética en la investigación científica que genera la universidad, así como los conocimientos y actitudes vinculados con la ética y bioética en la formación del odontólogo.

Se concluye que es un compromiso fomentar un cambio de paradigma, a través de un movimiento masivo que involucre los ámbitos familiar, profesional y académico, con el fin de integrar la reflexión ética en nuestro diario proceder.
\end{abstract}

Palabras clave: ética, bioética, formación del odontólogo

\section{ETHICS AND BIOETHICS INCLUSION IN GRADUATE AND POSTGRADUATE SURGEON- DENTIST CAREER IN PERU}

Abstract: This article focuses on the need to examine the graduate and postgraduate studies of the surgeon-dentist in Peru. The presence of an ethic normative in scientific investigation generated by the University is analysed from the training of the professor's point of view. The paper also focuses on the knowledge and attitudes entailed with ethics and bioethics in the odontologist's shaping.

The paper deduces that a change of paradigm is an engagement that has to be encouraged through a massive movement that should involve family and academic contours aiming at integrating ethical refection in our daily proceedings.

Key words: ethics, bioethics, odontologist's formation

\section{INCLUSÃO DA ÉTICA E BIOÉTICA NA FORMAÇÃO DE GRADUAÇÃO E PÓS-GRADUAÇÃO DO CIRURGIÁO DENTISTA NO PERU}

Resumo: Revisam-se aspectos da inclusão da ética e bioética na formação de graduação e pós-graduação do cirurgiâo dentista no Peru. Do ponto de vista da formação do docente, analisa-se a presença de normatividade ética na pesquisa científica que a universidade gera, assim como os conhecimentos e atitudes vinculados à ética e bioética na formação do odontólogo. $\mathrm{O}$ artigo conclui que é um compromisso fomentar uma mudança de paradigma, através de um movimento massivo que envolva os âmbitos familiar, profissional e acadêmico, com o objetivo de integrar a reflexão ética no trabalho diário.

Palavras chave: ética, bioética, formação do odontólogo

Cirujano-Dentista, Magíster en Estomatología, Universidad Peruana Cayetano Heredia, Perú

Correspondencia: 08733@upch.edu.pe 


\section{Introducción}

Muchos aspectos saltan a la vista si queremos referirnos al tema de la ética en la formación universitaria del odontólogo: por ejemplo, la imagen que debe proyectar el docente al dictar una clase, la preparación de los programas educativos sobre la base de valores en los planes curriculares de pre y posgrado, la normatividad ética en la investigación científica o, tal vez, las actitudes éticas en el quehacer diario del profesional o del estudiante.

El docente universitario tiene una función fundamental en el desarrollo de proyectos educativos que faciliten el aprendizaje y en propiciar influencias positivas conducentes a la formación y desarrollo de valores en los estudiantes. Por lo tanto, "debiera ser un modelo educativo en virtud de sus cualidades personales y profesionales" $(1,2)$. Entonces, es necesario analizar si esta condición se presenta realmente, si nuestras instituciones educativas preparan un personal docente que cumpla con esta misión y si la selección de este recurso humano es la más adecuada.

En la mayoría de las facultades se capacita al docente en conocimientos actualizados en odontología y en técnicas didácticas. Sin embargo, es difícil encontrar talleres sobre temas de socialización para una mejor comunicación docente-alumno, aplicación y discusión de criterios éticos en la práctica profesional o aspectos bioéticos en la investigación científica, entre otros.

Cabe preguntarnos si estamos preparados, como docentes, para orientar correctamente al alumno en su práctica diaria, en la cual deberá enfrentar nuevos problemas sociales, profesionales y éticos. Es pues inminente un cambio de paradigma, para que el profesor integre la enseñanza de conocimientos y destrezas en odontología con motivación hacia la autocrítica, trabajo en equipo y reflexión en dilemas éticos.

\section{Situación académica actual}

Con relación a los planes de estudio, es poco común encontrar cursos de ética o de bioética presentes en los planes curriculares de odontología. Tradicionalmente, se han incluido cursos de Odontología Legal y Deontología; es allí donde se incorpora información de aspectos relacionados con los códigos de ética odontológicos. En otros casos, se incluye información ética y bioética como capítulos inmersos en otras asignaturas, generalmente desde el punto de vista social de la atención dental y, asimismo, en la relación odontólogo-paciente.

Según Otero(3), hasta 2004 las facultades de odontología en el Perú sumaban veintiuna. En éstas se incluye el curso de Odontología Legal en el cuarto o quinto año de estudios, a excepción de nuevas escuelas que incorporan el curso de ética o bioética en su plan de estudios. En otros casos, comprenden contenidos de códigos de ética y principios de bioética en cursos relacionados.

Como menciona Pérez, en un trabajo de investigación realizado en la Escuela Nacional de Salud Pública de $\mathrm{Cuba}(4)$, la presencia de la ética en el proceso de formación del magíster en salud es limitada, lo que se manifiesta en el nulo o reducido espacio que ocupan sus contenidos en los programas impartidos y en la baja frecuencia con que se presentan las sustentaciones éticas explícitas en los trabajos desarrollados. Evangelista(5) revisó los temas de investigación de tesis de maestría en estomatología en una universidad particular en Lima, encontrando que no es de interés de los egresados enfocarse en temas de ética o bioética.

Asimismo, la normatividad ética en la investigación científica en pre y posgrado es un aspecto poco tratado. Las universidades cuentan con diferentes modalidades para obtención de título, pero la más empleada es el desarrollo de una tesis. Por otro lado, en estudios de maestría y doctorado es imprescindible elaborar y desarrollar una investigación científica, ya que uno de los pilares de la educación superior universitaria es la producción de conocimientos.

\section{Desafíos}

Es altamente razonable que la formación que entregan las facultades de odontología incluya conocimientos y desarrollo de habilidades clínicas, ampliamente detalladas en el currículo; sin embargo, es necesario analizar también los intereses y valores que más tarde regirán y serán la característica fundamental del desempeño profesional del odontólogo, como la honestidad y la responsabilidad de sus actos, reflejo de un profesional integralmente competente. Estos rasgos marcarán la diferencia entre los egresados pues, aunque todos obtienen conocimientos actualizados y destrezas clínicas en odontología, tanto en el pre como en el posgrado, lo que caracteriza a un profesional formado integralmente es su proceder y la correcta toma de decisiones, esto es, 
la reflexión crítica sobre aspectos morales involucrados en las decisiones biomédicas.

Es relevante incluir la formación de valores en el plan de estudios, aunque esto no debe limitarse a un proceso abstracto de transmisión de información, en el cual el estudiante sea un ente pasivo en la recepción del mensaje, sino que éste debe asumir una posición activa en la apropiación de la información para la construcción de sus propios valores. Para ello es fundamental el enfoque motivador y orientador del docente y del entorno académico.

Los valores, como formaciones motivacionales de la personalidad, se construyen y desarrollan a lo largo de toda la vida del ser humano, en un complejo proceso en el que intervienen la familia, la escuela y la sociedad. Es en la universidad donde el estudiante recién ingresado va alcanzando el desarrollo de su personalidad: consolida conocimientos con actitudes que orientan su actuación, regulando sus actos y alcanzando expresiones de autodeterminación, enfrentando sus prácticas preprofesionales y la problemática del entorno. Por esta razón, se considera que la educación en valores adquiere en este período de la vida una importancia extraordinaria, ya que existen mayores posibilidades para su consolidación, con perspectiva inmediata hacia la reflexión personalizada, congruencia y perseverancia en la regulación de la conducta(6).

En toda investigación en que interviene como objeto un ser humano surge generalmente un conflicto(7), puesto que, muchas veces, el investigador prioriza los objetivos del estudio, convirtiendo al sujeto de investigación sólo en un medio para alcanzar sus resultados. La utilización de seres humanos en investigación no es un asunto nuevo. El desarrollo científico-tecnológico ha puesto a los estudiantes y profesionales de salud -en este caso de odontología- frente a un dilema entre tecnología y valores.

La universidad debe educar a los futuros profesionales de la salud en principios que los lleven a respaldar el derecho universal del hombre a la salud y el respeto a su integridad en el marco de la investigación científi$\mathrm{ca}(8,9)$. Los estudios llevados a cabo por estudiantes de pre y posgrado de estomatología se deben regir por normas y principios éticos nacionales e internacionales; sin embargo, la falta de formación ética ha originado deficiencias en su aplicación. Es común observar en los protocolos de investigación y más tarde en las tesis concluidas que los estudiantes no han considerado aspectos bioéticos en el desarrollo de sus proyectos.

Son escasos los comités de ética en el ámbito de las instituciones, por lo que no se considera la inscripción previa de la investigación e, incluso, no siempre se solicita el consentimiento informado, aun cuando es inevitable ya que el estudio se realiza en seres humanos. Con relación a la función de estos comités, Lecca y colaboradores (10) refieren que, si bien los comités de ética de la investigación (CEI) peruanos cumplen con los requisitos de estructura, la mayoría presenta deficiencias y problemas para su funcionamiento, por lo que es necesario establecer políticas locales que fortalezcan estas instancias de protección en Perú.

La falta de normas en las direcciones de grado de las facultades y el poco conocimiento de este tema entre la mayoría de los asesores que conducen al alumno originan que este problema se agrave. Roberto Llanos sostiene que, en la medida en que los líderes reciban información académica, se garantiza la posibilidad de iniciar y mantener comunicación de buena calidad en este tema(11).

En relación con las actitudes, $\mathrm{Newcomb}$ (12) señala que los seres humanos adquieren actitudes a través de los procesos de interacción recíproca. Whittaker (1987), Baron y Byrne coinciden con Newcomb en que la principal fuente por la que se adquieren las actitudes, preferencias, creencias y tendencias del comportamiento es la interacción con las demás personas.

En el caso de los niños, las personas que refuerzan actitudes positivas son los padres, otros parientes o los maestros. Entre los adultos, las personas clave pueden ser los representantes de la autoridad, los compañeros de trabajo o los miembros de los grupos a los que el individuo quisiera pertenecer. De ahí la importancia de asistir a conferencias o participar activamente en foros, creando un entorno de discusión relacionado con el área de la bioética, lo cual les permitirá adoptar y reforzar actitudes cuyas consecuencias sean positivas.

En nuestro medio, la Asociación Peruana de Bioética cumple de manera significativa esta función, propiciando reuniones multidisciplinarias mensuales.

\section{Conclusiones}

Es necesario brindar información acerca de principios y normas éticas al estudiante de odontología, de tal 
forma que se involucre en la reflexión de los dilemas que puede enfrentar en el desarrollo de su vida profesional, resaltando la importancia de interiorizar valores de responsabilidad, honestidad y justicia como primordiales en la relación odontólogo-paciente.
Debe ser un compromiso de quienes estamos relacionados con este tema fomentar un cambio de paradigma, a través de un movimiento masivo que involucre distintos ámbitos, como el familiar, profesional y académico, con el objetivo de incrementar los conocimientos y generar actitudes positivas hacia la bioética en nuestro diario proceder.

\section{Referencias}

1. Arellano M. El proceso de enseñanza y aprendizaje de la Bioética. Revista CONAMED 2003; 8(2): 5-13.

2. Barreiro H, González J, Basanta M, Barreiro A. Criterios sobre la enseñanza de pregrado de la asignatura Medicina Legaly Ética Médica. La Habana: Instituto Superior de Ciencias Médicas de La Habana, Instituto de Medicina Legal; 2001

3. Otero J, Otero J. La paradoja de la Odontología. Odontología, Ejercicio Profesional 2004;5(10) (Sitio en Internet) Disponible en: http://www.odontomarketing.com/edit54oct2004.htm Último Acceso el 28 de abril de 2008.

4. Pérez CM. La Ética en la formación del Máster en Salud Pública. Rev Cubana Educ Med Super 2002; 16(3): 211 220.

5. Evangelista A. Trabajos de investigación realizados en los talleres de tesis del Centro Multidisciplinario de Investigación en Salud Oral de la Facultad de Estomatología de la Universidad Peruana Cayetano Heredia desde 1995 al 2002. Tesis para optar Grado de Magíster en Estomatología. Lima: Facultad de Estomatología de la Universidad Peruana Cayetano Heredia; 2003

6. Pernas M, Ortiz M, Menéndez A. Consideraciones sobre la formación ética de los estudiantes de ciencias médicas. Rev Cubana Educ Med Super 2002; 16(2): 113-119.

7. Lolas F, Quezada A, Rodríguez E. Investigación en Salud. Dimensión Ética. Santiago de Chile: CIEB Universidad de Chile; 2006.

8. Rooff S, Preece P. Helping medical students to find their moral compasses:ethics teaching for second and third year undergraduates. J Med Ethics 2004; 30: 487-489.

9. Rodríguez de Romo AC. Utopía o realidad: ¿`iene sentido enseñar ética médica a los estudiantes de medicina? An Med Assoc Med Hosp. ABC 2000; 45(1): 45-50.

10. Lecca L, Llanos-Zavalaga F, Ygnacio E. Características de los Comités de Ética en Investigación del Perú autorizados para aprobar ensayos clínicos. Rev Med Hered 2005; 16: 3-10.

11. Pessini L, Barchifontaine C, Lolas F. Perspectivas de la Bioética en Iberoamérica. Santiago de Chile: CIEB Universidad de Chile; 2007.

12. Newcomb T. Manual de Psicología Social. Tomo 1. Buenos Aires: Eudeba; 1963.

Recibido: 3 de abril de 2008

Aceptado: 25 de abril de 2008 